\title{
Erratum to: Appraising the Role of Iron in Brain Aging and Cognition: Promises and Limitations of MRI Methods
}

\author{
Ana M. Daugherty ${ }^{1} \cdot$ Naftali Raz $^{1,2}$
}

Published online: 4 September 2015

(C) Springer Science+Business Media New York 2015

Erratum to: Neuropsychol Rev

DOI 10.1007/s11065-015-9292-y

Under the heading "Myelin Biases In Vivo Estimates of Iron," fourth sentence, the word "inflates" should be changed to "biases." The correct sentence is shown below:

Presence of myelin biases the estimates of iron content that are derived from R2* (Glasser and Van Essen 2011; Haacke et al. 2005), phase and QSM/SWIM (Bilgic et al. 2012; Deistung et al. 2013; Langkammer et al. 2012a, b; Wisnieff et al. 2014) and the bias may be the strongest in myelin-rich regions.

The online version of the original article can be found at http://dx.doi.org/ 10.1007/s11065-015-9292-y.

$\triangle$ Naftali Raz

nraz@wayne.edu

Institute of Gerontology, Wayne State University, 87 E. Ferry St., 226

Knapp Bldg., Detroit, MI 48202, USA

2 Department of Psychology, Wayne State University,

Detroit, MI 48202, USA 